\title{
Clostridium difficile and inflammatory bowel disease: implications for current clinical practice Venkataraman Subramanian
}

\author{
Address: Nottingham Digestive Diseases Centre and Biomedical Research Unit, Nottingham University Hospital (QMC campus), \\ Nottingham NG7 2UH, UK \\ Email: venkat@nottingham.ac.uk
}

Fl000 Medicine Reports 2009, I:25 (doi: 10.3410/MI-25)

The electronic version of this article is the complete one and can be found at: http://FI000.com/Reports/Medicine/content///25

\begin{abstract}
Clostridium difficile infection should always be included in the differential diagnosis of patients with refractory inflammatory bowel disease (IBD) symptoms, as it is well known to induce or mimic a flare of IBD. There is currently an alarming increase in the incidence of $C$. difficile infection in Europe and North America. Current epidemiologic data suggest that more virulent strains, such as $C$. difficile 027/ $\mathrm{NAPI} / \mathrm{BI}$, are emerging. Testing for both $C$. difficile toxins $A$ and $B$ should be done, and often serial stool samples may be required, especially if the index of suspicion is high. Patients with IBD do not necessarily require a history of antibiotic use or hospitalization to acquire the infection and most do not have pseudomembranes on sigmoidoscopy. C. difficile infection is associated with longer hospital stays, increased mortality, and higher complication rates, including colectomy. Treatment with oral vancomycin is probably the drug of choice as one study suggests that patients had lower colectomy rates on this drug. Further work is required to clarify the best management strategies for $C$. difficile in IBD patients, and more effort is needed to halt the rising incidence of this infection, perhaps with adherence to safer antibiotic prescribing guidelines, hand washing, and cleaner hospital environments, which should reduce the risk of acquiring and spreading this preventable infection.
\end{abstract}

\section{Introduction and context}

Issues in Clostridium difficile infection and inflammatory bowel disease

Clostridium difficile infection has gained recent attention because of its rising incidence in hospitalized patients. $C$. difficile complicates the management of inflammatory bowel disease (IBD) and often can be overlooked in a patient who presents with an exacerbation of symptoms. The dilemma facing physicians is when, how often, and in whom to order expensive stool tests for $C$. difficile. The other issue is how to differentiate faecal carriage of $C$. difficile from an infection in patients with IBD. The association of $C$. difficile with IBD is neither novel nor recent. In a series of case reports in the early 1980s, LaMont and Trnka [1] identified an association between the presence of $C$. difficile toxin and IBD exacerbation. Subsequently, a prospective study by the same investigators showed that $C$. difficile toxin was found in the stool of $19 \%$ of IBD patients, suggesting that $C$. difficile can cause worsening of disease activity in patients with IBD [2]. In that study, C. difficile was not necessarily associated with antibiotic use. In contrast, a second group of investigators found that $C$. difficile toxin was present mostly in the stool of IBD patients exposed to antibiotics, and concluded that there was no association with IBD activity [3]. More recently, Meyer and colleagues [4] showed that $20 \%$ of patients with IBD who relapsed had a positive stool test for $C$. difficile toxin. C. difficile was observed in 10 of 12 patients whose stool specimens were positive for any pathogen, all 10 of whom improved clinically with treatment for $C$. difficile. Furthermore, antibiotic use was associated with $90 \%$ of the patients who tested positive for C. difficile [4]. 
What has changed since these early reports is the rising incidence of $C$. difficile infections in hospitalized patients in North America [5,6] and Europe [6] noted mostly since the turn of this century. Data from US hospital discharges have revealed a doubling of C. difficileassociated disease (CDAD) between 1996 and 2003 [7], and the proportion of patients diagnosed with $C$. difficile increased by $26 \%$ from 2000 to 2001 in nonfederal US hospitals [8]. Patients recently treated with broad-spectrum antibiotics, hospitalized patients, immunocompromised individuals, the elderly, and patients with cancer are all at increased risk for CDAD. IBD patients are frequently on long-term immunosuppressants, are more likely to be hospitalized, often require antibiotics, and have many of the risk factors for acquiring CDAD. This brief report will look at the impact of rising C. difficile rates on the IBD population and the implications for the clinical management of these patients in this era of epidemic rates of C. difficile infections.

\section{Recent advances}

\section{C. difficile is an escalating problem in IBD patients}

Data from two tertiary referral centres from the US showed that the incidence of CDAD has not only risen among hospitalized patients, but was disproportionately higher in those with IBD $[9,10]$. A study from St Louis, Missouri, examined all admissions to a tertiary medical centre from 1998 to 2004 and found that the incidence of C. difficile infection had increased among both IBD and non-IBD patients, with the greatest increase occurring among those with ulcerative colitis (UC) [10]. The odds ratio for CDAD in Crohn's disease (CD) was 2.1 [95\% confidence interval (CI) 1.3 to 3.4], and the odds ratio for CDAD in UC was 4.0 (95\% CI 2.4 to 6.6). The median numbers of days from admission to acquiring CDAD were 0.5 days for $\mathrm{UC}, 0.8$ days for $\mathrm{CD}$, and 4.0 days for all non-IBD diagnoses. While $63 \%$ of IBD patients who acquired CDAD did so within 48 hours of admission, $63 \%$ of non-IBD patients did so only after 48 hours [10].

Another study, from Milwaukee, Wisconsin, reviewed data from all patients who were followed at this tertiary IBD centre between 2000 and 2005 [9]. During the last 2 years of the study, the incidence of $C$. difficile infection rose from 1.8 to $4.6 \%$. The proportions of IBD patients within the total number of patients diagnosed with CDAD were $4 \%$ in 2003, 7\% in 2004, and 16\% in 2005. Of these, $76 \%$ of individuals with IBD contracted CDAD as an outpatient and $61 \%$ of IBD patients with $C$. difficile infection had prior antibiotic exposure (most commonly, ciprofloxacin). Community-acquired CDAD occurred three times as often as nosocomial disease.
None of the IBD patients with CDAD had pseudomembranes [9]. Also, data from Leuven, Belgium, have shown a 3.75-fold increase in CDAD between January 2000 and January 2008 [11]. Considerably fewer IBD patients $(42 \%)$ had been noted to have taken prior antibiotics compared with non-IBD patients (69\%).

\section{Impact of C. difficile infection on IBD outcomes}

In the Milwaukee study, more than $50 \%$ of patients with IBD and C. difficile required hospitalization and $20 \%$ of hospitalized IBD patients with CDAD required colectomy. Immunosuppression and colonic involvement were independent risk factors for CDAD in IBD [9]. Furthermore, US data from the Nationwide Inpatient Sample (2003) suggested that patients with C. difficile and IBD had four times the mortality of those admitted with IBD alone [adjusted odds ratio (aOR) 4.7, 95\% CI 2.9 to 7.9 ] and twice the mortality of those admitted with C. difficile but not IBD (aOR 2.2, 95\% CI 1.4 to 3.4) [12]. IBD patients with $C$. difficile also stayed in hospital longer by an average of 3 days ( $95 \%$ CI 2.3 to 3.7 days).

Intriguingly, the clinical outcomes in the study from Leuven were better, with IBD patients having a shorter duration of hospitalization and with only one patient with UC needing a colectomy [11]. Similarly, the St Louis, Missouri group also noted no differences in length of stay between CDAD patients with IBD and those without IBD [10].

\section{Unusual aspects ofC. difficile infection in IBD}

Several factors may underlie the increasing incidence of C. difficile among patients with IBD. Increased awareness among physicians, more sensitive enzyme-linked immunosorbent assay tests for detection of the toxin in stool samples, and a serendipitous rise unrelated to IBD in hospitalized patients have all been put forward as explanations. However, there seem to be many differences between IBD patients infected with $C$. difficile compared with others. The first is that a relatively larger number of IBD patients have community-acquired infections $[9,10]$. Second, fewer IBD patients have had prior antibiotic use [9-11]. Third, none of the patients with IBD appear to have developed pseudomembranes $[9,13]$. The absence of pseudomembranes can be attributed to immunosuppression, as studies suggest that patients with bone marrow or stem cell transplants on immunosuppression, and those with UC on steroids, did not develop pseudomembranes with $C$. difficile infection [13]. Finally, there have been increasing reports of C. difficile enteritis complications in patients after recent total colectomy [14], or even, rarely, many years following a protocolectomy, usually presenting as increased ileostomy output or prolonged ileus [15]. 
While C. difficile enteritis is unusual, it may not be surprising as early studies involving autopsies [16] and jejunal aspirates [17] have suggested that the small bowel may in fact be the reservoir for $C$. difficile in humans.

There is considerable debate on whether $C$. difficile is just a colonizer in most patients with IBD or whether the infection does indeed cause an infective colitis superimposed on IBD. An intriguing report from Southern India (where C. difficile infection is still an uncommon cause of antibiotic-associated diarrhoea) using quantitative polymerase chain reaction (PCR) of species-specific 16s rDNA of C. difficile detected C. difficile in 34 of 37 UC patients and 20 of 36 healthy volunteers [18]. However, there were significantly higher numbers of $C$. difficile in UC patients but only eight UC and two healthy volunteers were toxin-positive as well. None of the patients had received antibiotics for at least 2 months prior to the study, and none of the patients were treated for $C$. difficile infection with no adverse events [18]. This suggests that there is an overgrowth of $C$. difficile in UC patients in India detected by PCR, but the clinical significance is uncertain.

\section{Implications for clinical practice Top tips for management}

1. Always think C. difficile Have a high index of suspicion for $C$. difficile in symptomatic IBD patients. Patients with unusual symptoms like watery rather than bloody diarrhoea in combination with colonic IBD, fever, nonresolving symptoms with standard treatment, and the presence of risk factors like recent antibiotic use or patients on maintenance immunosuppression should have their stool checked for $C$. difficile and treated if positive.

2. If in doubt, test and test again. Testing multiple stool samples may be needed to confirm the diagnosis. Ninety-two percent of patients were detected by the fourth sample in the Milwaukee study, and in one patient the diagnosis was made on only the eighth sample [9].

3. Never underestimate your foe. Due to concomitant IBD, it would be difficult to grade the severity of the $C$. difficile colitis but it would be prudent to treat these patients as if they had severe CDAD. The Milwaukee experience suggests a high rate of failure with metronidazole and a lower rate of colectomy with oral vancomycin, therefore oral vancomycin should be the preferred drug of choice [9].
4. Expect the unexpected. Consider C. difficile in patients with prolonged ileus post-colectomy for IBD or increased ileostomy outputs. Chronic pouchitis has also been attributed to C. difficile in patients with ileo anal pouch anastomosis [19].

5. Prevention is cure. Concomitant C. difficile and IBD has been associated with higher mortality, higher colectomy rates, and longer hospitalization. We, as the medical fraternity, have a responsibility to our patients and should not be lax in adhering to safe antibiotic prescribing guidelines or in implementing simple measures like hand washing between patients to reduce the spread of this infection.

\section{Future directions}

Randomized controlled trials are needed to clarify the best management strategy for concomitant $C$. difficile in IBD patients. What dose of vancomycin should be used? Would a tapering schedule of vancomycin help prevent relapse? Are probiotics of any use to prevent or treat this infection in IBD patients? How does one differentiate colonization from infection in patients with already diseased colons? Finally, as already mentioned, a concerted effort is needed to reduce the rising tide of $C$. difficile worldwide with greater emphasis on safer antibiotic prescribing guidelines, hand washing, cleaner hospital environments, and greater physician and community awareness. Until this epidemic is controlled, we will always have tough questions to answer in the management of these seriously ill patients on a daily basis.

\section{Abbreviations}

aOR, adjusted odds ratio; CD, Crohn's disease; CDAD, Clostridium difficile-associated disease; CI, confidence interval; IBD, inflammatory bowel disease; PCR, polymerase chain reaction; UC, ulcerative colitis.

\section{Competing interests}

The author declares that he has no competing interests.

\section{References}

I. Lamont JT, Trnka YM: Therapeutic implications of Clostridium difficile toxin during relapse of chronic inflammatory bowel disease. Lancet 1980, I:38I-3.

2. Trnka YM, Lamont JT: Association of Clostridium difficile toxin with symptomatic relapse of chronic inflammatory bowel disease. Gastroenterology 1981, 80:693-6.

3. Meyers S, Mayer L, Bottone E, Desmond E, Janowitz HD: Occurrence of Clostridium difficile toxin during the course of inflammatory bowel disease. Gastroenterology 1981, 80:697-700.

4. Meyer AM, Ramzan NN, Loftus EV Jr., Heigh RI, Leighton JA: The diagnostic yield of stool pathogen studies during relapses of inflammatory bowel disease. J Clin Gastroenterol 2004, 38:772-5. 
5. McDonald LC, Killgore GE, Thompson A, Owens RC Jr, Kazakova SV, Sambol SP, Johnson S, Gerding DN: An epidemic, toxin genevariant strain of Clostridium difficile. N Engl J Med 2005, 353:2433-4I.

FI000 Factor 9.0 Exceptional

Evaluated by Jon Markowitz 16 Dec 2005

6. Warny M, Pepin J, Fang A, Killgore G, Thompson A, Brazier J, Frost E, McDonald LC: Toxin production by an emerging strain of Clostridium difficile associated with outbreaks of severe disease in North America and Europe. Lancet 2005, 366: 1079-84.

FI000 Factor 3.0 Recommended

Evaluated by Matthias Maiwald I4 Nov 2005

7. McDonald LC, Owings M, Jernigan DB: Clostridium difficile infection in patients discharged from US short-stay hospitals, 1996-2003. Emerg Infect Dis 2006, 12:409-15.

8. Dallal RM, Harbrecht BG, Boujoukas AJ, Sirio CA, Farkas LM, Lee KK, Simmons RL: Fulminant Clostridium difficile: an underappreciated and increasing cause of death and complications. Ann Surg 2002, 235:363-72.

9. Issa M, Vijayapal A, Graham MB, Beaulieu DB, Otterson MF, Lundeen S, Skaros S, Weber LR, Komorowski RA, Knox JF, Emmons J, Bajaj JS, Binion DG: Impact of Clostridium difficile on inflammatory bowel disease. Clin Gastroenterol Hepatol 2007, 5:345-5I.

10. Rodemann JF, Dubberke ER, Reske KA, Seo da H, Stone CD: Incidence of Clostridium difficile infection in inflammatory bowel disease. Clin Gastroenterol Hepatol 2007, 5:339-44.

II. Bossuyt P, Verhaegen J, Van Assche G, Rutgeerts P, Vermeire S: Increasing incidence of Clostridium difficile-associated diarrhea in inflammatory bowel disease. Journal of Crohn's and Colitis, in press.

12. Ananthakrishnan AN, McGinley EL, Binion DG: Excess hospitalisation burden associated with Clostridium difficile in patients with inflammatory bowel disease. Gut 2008, 57:205-I0.

13. Nomura K, Fujimoto $Y$, Yamashita M, Morimoto $Y$, Ohshiro M, Sato K, Oyake T, Kowata S, Konishi H, Yoshikawa T, Ishida Y, Taniwaki M; Japan Hematology/Oncology Study (J-HOST) Group Kyoto: Absence of pseudomembranes in Clostridium difficile-associated diarrhea in patients using immunosuppression agents. Scand J Gastroenterol 2009, 44:74-8.

14. Lundeen SJ, Otterson MF, Binion DG, Carman ET, Peppard WJ: Clostridium difficile enteritis: an early postoperative complication in inflammatory bowel disease patients after colectomy. J Gastrointest Surg 2007, I I:I 38-42.

15. Kim KA, Wry P, Hughes E Jr., Butcher J, Barbot D: Clostridium difficile small-bowel enteritis after total proctocolectomy: a rare but fatal, easily missed diagnosis. Report of a case. Dis Colon Rectum 2007, 50:920-3.

16. Taylor RH, Borriello SP, Taylor AJ: Isolation of Clostridium difficile from the small bowel. Br Med J (Clin Res Ed) I98I, 283:4I2.

17. Testore GP, Nardi F, Babudieri S, Giuliano M, Di Rosa R, Panichi G: Isolation of Clostridium difficile from human jejunum: identification of a reservoir for disease? J Clin Pathol 1986, 39:86I-2.

18. Balamurugan R, Balaji V, Ramakrishna BS: Estimation of faecal carriage of Clostridium difficile in patients with ulcerative colitis using real time polymerase chain reaction. Indian J Med Res 2008, I 27:472-7.

19. Shen B, Goldblum JR, Hull TL, Remzi FH, Bennett AE, Fazio VW: Clostridium difficile-associated pouchitis. Dig Dis Sci 2006, 5I:236I-4. 